\title{
The Effects of the Monoamine Stabilizer (-)-OSU6I62 on Binge-Like Eating and Cue-Controlled Food-Seeking Behavior in Rats
}

\author{
Kristin Feltmann ${ }^{*, 1,4}$, Chiara Giuliano ${ }^{2,4}$, Barry J Everitt' ${ }^{2}$ Pia Steensland' and Johan Alsiö ${ }^{2,3}$ \\ 'Centre for Psychiatry Research, Department of Clinical Neuroscience, Karolinska Institutet and Stockholm Health Care Services, Stockholm County \\ Council, Stockholm, Sweden; 'Department of Psychology and Behavioural and Clinical Neuroscience Institute, University of Cambridge, Cambridge, \\ UK; ${ }^{3}$ Department of Neuroscience, Unit of Functional Neurobiology, University of Uppsala, Uppsala, Sweden
}

\begin{abstract}
Binge-eating disorder (BED) is characterized by recurring episodes of excessive consumption of palatable food and an increased sensitivity to food cues. Patients with BED display an addiction-like symptomatology and the dopamine system might be a potential treatment target. The clinically safe monoamine stabilizer ( - )-OSU6I 62 (OSU6I62) restores dopaminergic dysfunction in long-term alcohol-drinking rats and shows promise as a novel treatment for alcohol use disorder. Here, the effects of OSU6I62 on consummatory (binge-like eating) and appetitive (cue-controlled seeking) behavior motivated by chocolate-flavored sucrose pellets were evaluated in non-food-restricted male Lister Hooded rats. OSU6I 62 significantly reduced binge-like intake of chocolate-flavored sucrose pellets without affecting prior chow intake. Furthermore, OSU6162 significantly reduced the cue-controlled seeking of chocolate-flavored sucrose pellets under a secondorder schedule of reinforcement before, but not after, the delivery and ingestion of reward, indicating a selective effect on incentive motivational processes. In contrast, the dopamine D2/D3 receptor antagonist raclopride reduced the seeking of chocolate-flavored sucrose pellets both pre- and post reward ingestion and also reduced responding under simpler schedules of seeking behavior. The DI/5 receptor antagonist $\mathrm{SCH} 23390$ had no effect on instrumental behavior under any reinforcement schedule tested. Finally, local administration of OSU6I62 into the nucleus accumbens core, but not dorsolateral striatum, selectively reduced cue-controlled sucrose seeking. In conclusion, the present results show that OSU6I62 reduces binge-like eating behavior and attenuates the impact of cues on seeking of palatable food. This indicates that OSU6I62 might serve as a novel BED medication.
\end{abstract}

Neuropsychopharmacology (2018) 43, 617-626; doi:I0.1038/npp.2017.215; published online 25 October 2017

\section{INTRODUCTION}

Binge-eating disorder (BED) is characterized by persistent uncontrollable episodes of eating large amounts of palatable food, and an increased responsivity to food-associated cues (Schienle et al, 2009) inducing food cravings and overeating (Curtis and Davis, 2014; Sobik et al, 2005). These behaviors resemble symptoms of substance use disorders, including loss of control over drug intake, drug cue sensitivity, and craving (Cassin and von Ranson, 2007). The mesocorticolimbic dopamine system may be implicated in the overlapping neural mechanisms between the two disorders, as well as the reinforcing effects of both palatable foods and drugs of abuse (Gonzales et al, 2004; Wise, 2006). In fact, intake of both palatable food and drugs of abuse (Ericson et al, 1998; Ito et al, 2000; Rada et al, 2005) and cues

*Correspondence: K Feltmann, Centre for Psychiatry Research, Norra Stationsgatan 69, plan 7, II 364 Stockholm, Sweden, Tel: +46 (0) 736353046, E-mail: Kristin.feltmann@ki.se

${ }^{4}$ These authors contributed equally to this work.

Received 12 June 2017; revised 28 August 2017; accepted 6 September 2017; accepted article preview online 12 September 2017 associated with these reinforcers (Di Ciano et al, 1998; du Hoffmann and Nicola, 2014) release dopamine in the nucleus accumbens (NAc) in rodents. Similarly, in both BED (Wang et al, 2011) and substance use disorders (Volkow et al, 2006), dopamine is released in the striatum following the presentation of food- and drug-associated cues, respectively. Moreover, like chronic drug intake, chronic binge-like intake of palatable food induces addictive-like behaviors and dopaminergic changes in the striatum (Avena, 2010; Naef et al, 2015), including decreased NAc dopamine D2 receptor levels in rodents (Avena, 2007; Bello and Hajnal, 2010; Colantuoni et al, 2001). In addition, genetic studies have associated binge-eating pathology in humans with polymorphisms of the dopamine transporter and D2 receptor (for review see Bello and Hajnal, 2010). Finally, the monoamine-releasing compound lisdexamfetamine is the only FDA-approved BED medication (Davis and Attia, 2017). However, because of the medication's side effects and abuse potential, improved treatment options are needed.

The monoamine stabilizer (-)-OSU6162 (OSU6162) (Sonesson et al, 1994) is a clinically safe compound (Johansson et al, 2012; Khemiri et al, 2015; Kloberg et al, 
2014) with the ability to increase and decrease dopaminergic activity depending on the prevailing dopaminergic tone (Rung et al, 2008; Tedroff et al, 1998). Instead of partial agonism, antagonism at both extrasynaptic dopamine D2 auto- and heteroreceptors (Tolboom et al, 2014) has been hypothesized to underlie this effect. We recently identified OSU6162 as a potential treatment for alcohol use disorder by showing that it attenuates alcohol-mediated behaviors (Steensland et $a l, 2012$ ) and restores striatal dopaminergic deficits (Feltmann et al, 2016) in alcohol-drinking rats, as well as attenuates alcohol craving in alcohol-dependent humans (Khemiri et al, 2015).

Based on the behavioral and neurochemical similarities between BED and substance use disorders, together with our observation that OSU6162 reduced sucrose drinking in rats (Steensland et al, 2012), we here explored the potential of OSU6162 as a novel BED treatment using relevant rodent models. The effect of OSU6162 on food consumption was investigated using a model of binge-like intake of highly palatable food (Cottone et al, 2008; Giuliano et al, 2012) and the ability of OSU6162 to affect the impact of food-associated cues was investigated using food seeking under a secondorder schedule of reinforcement (Giuliano et al, 2012). In the latter procedure, animals respond on a fixed-ratio schedule (FR10) for brief, contingent presentations of rewardassociated conditioned stimuli (CS), acting as conditioned reinforcers, during two $15 \mathrm{~min}$ intervals. Each interval is terminated by reward delivery (20 chocolate-flavored sucrose pellets). This procedure was used to investigate whether OSU6162, compared with dopamine D1 and D2 receptor antagonists, affects high-incentive food reward seeking directly (first interval, before reward delivery) or indirectly via alteration of the hedonic impact of food ingestion (second interval, after reward delivery) (Giuliano et al, 2012). Furthermore, to evaluate whether potential effects would be specific to prolonged seeking and CS responding, the effects of the compounds were also investigated under fixed interval (FI) schedules: one involving frequent reward delivery ( 1 min, FI1) and one similar to the second-order schedule but without contingent CS presentations (FI15). Finally, to identify brain areas involved in mediating the systemic effects of OSU6162 on the second-order schedule, local bilateral OSU6162 infusions were given into the NAc core and dorsolateral striatum (DLS). These two structures were targeted as they, in contrast to the NAc shell, have been consistently shown to be implicated in conditioned reinforcement and in the effects of conditioned reinforcers on instrumental seeking behavior in a second-order schedule of reinforcement (Ito et al, 2000); for example, the integrity of the NAc core is necessary for the expression of conditioned reinforcement and the acquisition of a novel response for a food- or cocaine-paired conditioned reinforcer (Di Ciano, 2008; Di Ciano and Everitt, 2004; Parkinson et al, 1999).

\section{MATERIALS AND METHODS}

Detailed descriptions of animals (male Lister Hooded rats (Charles River, Margate, UK)), housing conditions, intracranial guide cannula implantation and drug infusion, histological assessment of cannula placement, and drugs and chemicals are available in Supplementary Information.
All procedures were conducted according to the UK Animal (Scientific Procedures) Act 1986 Amendment Regulations 2012, following ethical review by the University of Cambridge Animal Welfare and Ethical Review Body (AWERB).

\section{Binge-Like Eating of Highly Palatable Food}

Paradigm. The binge-eating procedure was used to induce a binge-like hyperphagia of palatable food and a prior chow hypophagia in anticipation of the palatable food (negative anticipatory contrast).

The procedure was performed as described previously (Cottone et al, 2008; Giuliano et al, 2012) but minor modifications were made. In brief, male rats with free access to rodent chow in the home cage throughout the experiment were transferred daily to behavioral chambers. After $2 \mathrm{~h}$ of food deprivation, rats had access to chow in one (first) and then another (second) feeder for $10 \mathrm{~min}$ in each feeder. After 20 sessions rats were divided into two groups, both of which had access to chow from the first feeder and chocolateflavored sucrose pellets (chow/sucrose group, $n=10$ ) or chow (chow/chow group, $n=10$ ) from the second feeder, respectively (Figure 1a).

Treatment. To ensure binge-like eating in the chow/ sucrose group, testing began at least 15 sessions after the chocolate-flavored sucrose pellets had been introduced in the second feeder (Figure 1a). Thereafter, OSU6162 (5, 10, 15, and $30 \mathrm{mg} / \mathrm{kg}$, subcutaneously (i.p.)) or vehicle were administered $60 \mathrm{~min}$ before giving access to the first feeder using a Latin square design with 2 days of re-baselining in between injections. One rat in the binge-eating paradigm was excluded from analysis because of ill health.

\section{Seeking of Highly Palatable Food: Second-Order Schedule of Reinforcement}

Paradigm. The second-order schedule of high-incentive food reinforcement procedure was performed as previously described (Giuliano et al, 2012), except that rats in the present experiment were given unlimited access to food in the home cages throughout the experiment. Briefly, 24 rats were initially trained in operant chambers to press a lever under a FR1 schedule for delivery of chocolate-flavored sucrose pellets, paired with a $20 \mathrm{~s}$ light stimulus (CS). Following five sessions on FR1 (Figure 1b), sessions under FI schedules were introduced during which pellet delivery and $20 \mathrm{~s}$ CS presentation was restricted to the first lever press after the end of each 1 min interval (FI1). After $\sim 15$ sessions on FI1, the length of the intervals was progressively increased up to $15 \mathrm{~min}$ (FI15) during six sessions (FI2, FI4, FI6, FI8, FI10, and FI15), whereas the maximum total number of 40 pellets/session remained constant (ie, during a FI15 session, 20 pellets were delivered together with a $20 \mathrm{~s} \mathrm{CS}$ at the end of each of the two intervals). Following 13 sessions on FI15, a second-order schedule of reinforcement was introduced, differing only from the FI15 schedule in the presentation of a brief $1 \mathrm{~s}$ CS together with every tenth lever press during each interval. Pellet consumption was assessed by checking for any remaining pellets in the food tray at the end of each session. 
a Binge-eating model

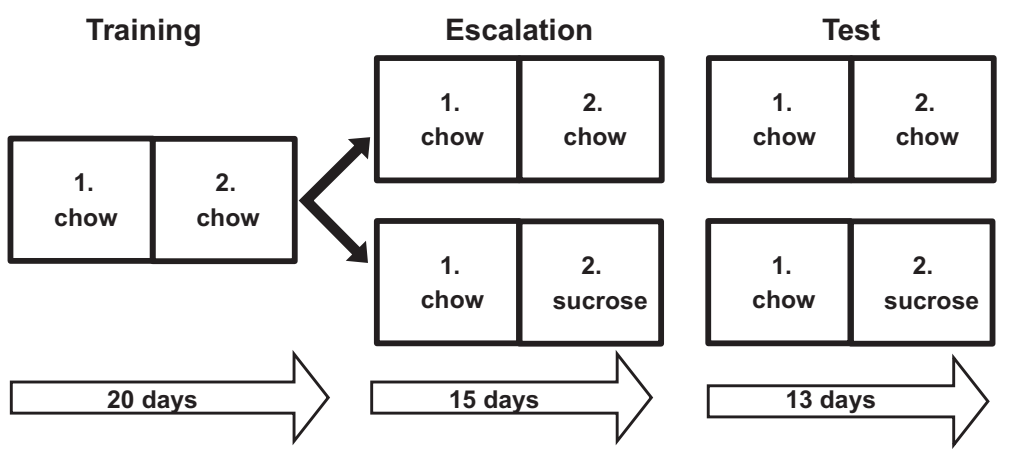

b Second-order of reinforcement

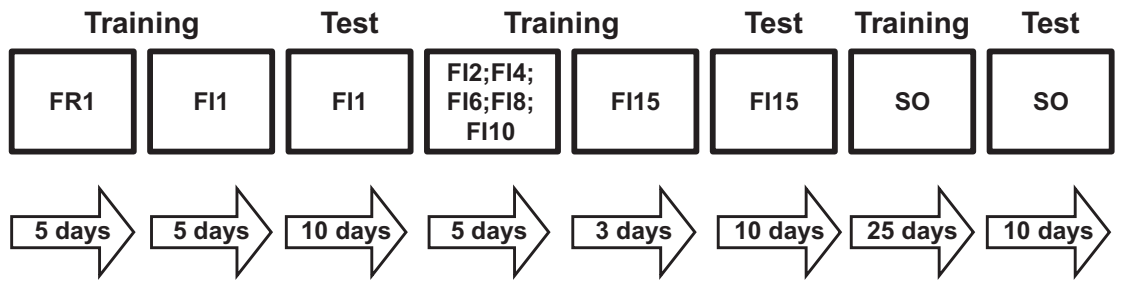

C Local infusions for second-order of reinforcement

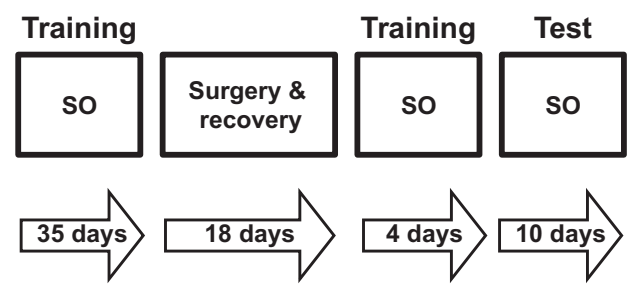

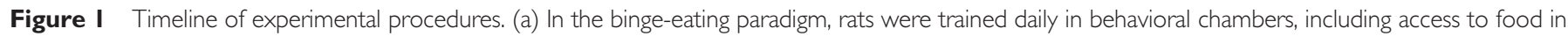

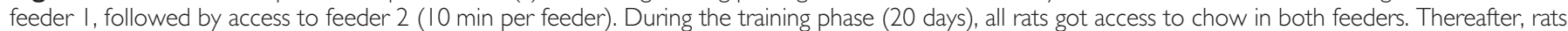

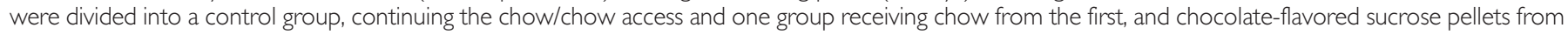

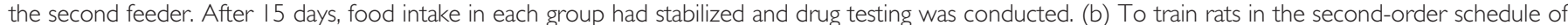

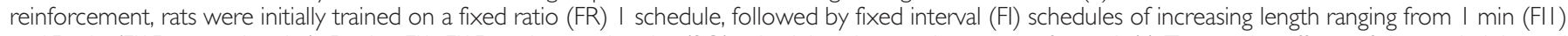

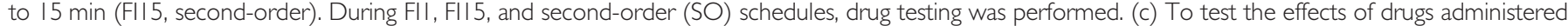

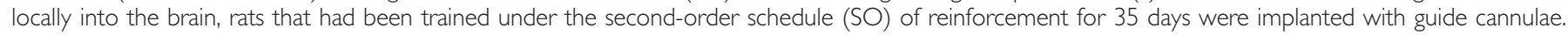

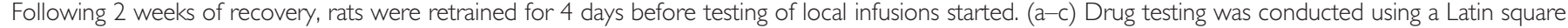
design, ie, each rat received each dose, with 2 days of rebaselining in between injections. The period of each training and testing stage is indicated.

Treatment. The animals were divided into three groups (matched for baseline FI1 responding, $n=8$ per group) and treated with OSU6162 $(5,10$, and $15 \mathrm{mg} / \mathrm{kg}$, s.c.), raclopride (0.01, 0.03 , and $0.1 \mathrm{mg} / \mathrm{kg}$, s.c.), or SCH23390 (0.003, 0.01, and $0.03 \mathrm{mg} / \mathrm{kg}$, intraperitoneally (i.p.)), respectively. Testing was conducted within each treatment group after reaching stable baseline performance on FI1, FI15, and second-order schedules, respectively, using a Latin square design within each schedule with at least 2 days of rebaselining in between injections.

Surgical procedures and intracerebral infusions. Two cohorts of rats trained on the second-order schedule of reinforcement (Figure 1c) were implanted bilaterally with guide cannulae above the NAc core $(\mathrm{AP}+1.7, \mathrm{ML} \pm 1.9$, $\mathrm{DV}-1.5)$ or DLS (AP +1.2, ML $\pm 3.0, \mathrm{DV}-2.0)$. The first cohort was the same as in the previous experiment (systemic injections of OSU6162, raclopride, or
SCH23390); surgery took place within 2 weeks after the last injection on the second-order schedule. The second group was prepared using a similar training procedure with the exception of shorter FI1 and FI15 schedules as no drug testing was conducted.

OSU6162 $(0.5,1.5$, and $5 \mu \mathrm{g} / 0.5 \mu \mathrm{l} /$ side, Latin square design) was bilaterally infused into the NAc core or DLS $5 \mathrm{~min}$ before the start of the session. Only animals with cannula placements in the target areas were included in the statistical analysis (see details in Supplementary Information).

\section{Statistical Analysis}

To identify overall effects on acquisition of the binge-like eating behavior, food intake (kcal) was analyzed using repeated-measures analysis of variance (ANOVA) with diet (group: chow/chow, chow/sucrose) as the between-subject 
Chow/Chow Group
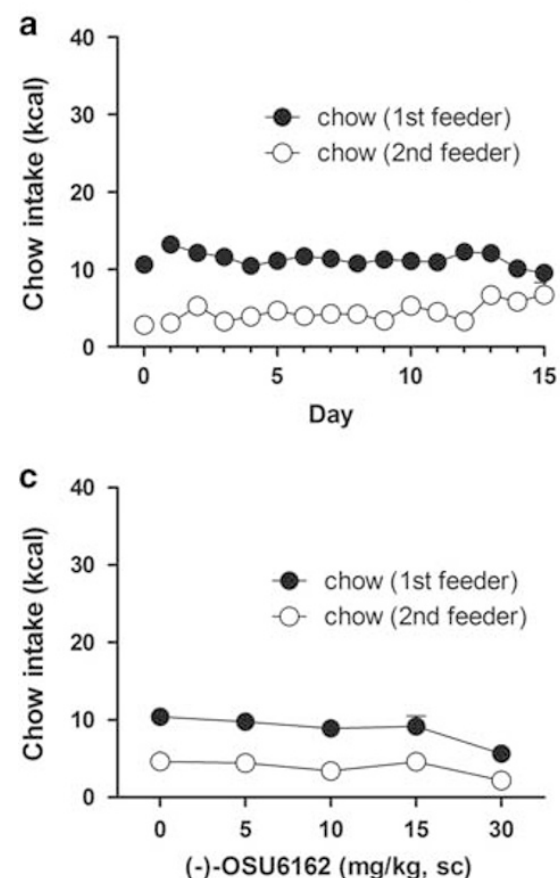

Chow/Sucrose Group
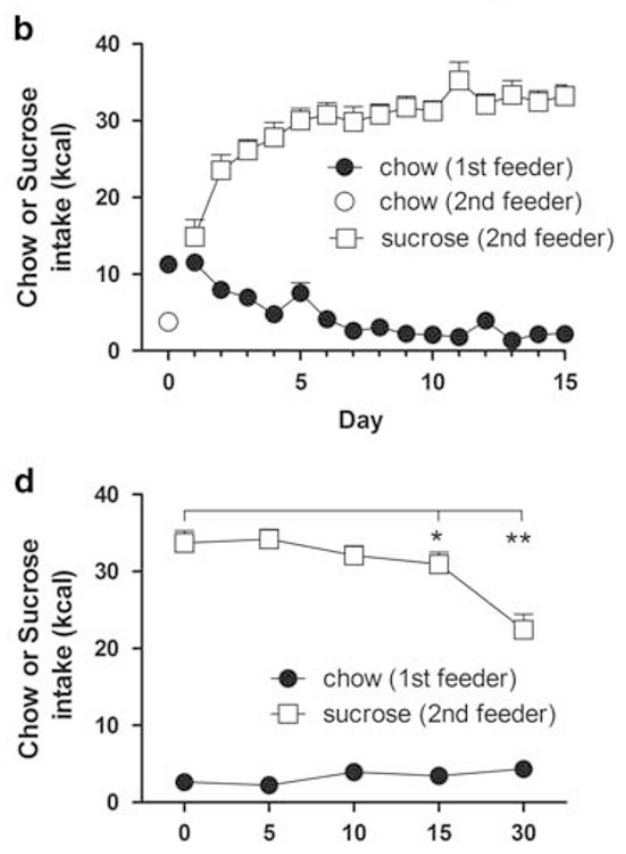

(-)-OSU6162 (mg/kg, sc)

Figure 2 OSU6I 62 reduces intake of highly palatable food in a binge-like eating paradigm. The binge-eating procedure used consisted of three contiguous phases: $2 \mathrm{~h}$ food deprivation, first feeder: 10 min access to chow (closed circles), second feeder: 10 min access to chow (open circles) or chocolate-flavored sucrose pellets (open squares). Values represent the mean \pm SEM food intakes in kcal ( $n=9-10 / g r o u p)$. The chow/chow-fed rats (a) displayed stable intake of chow over 15 sessions. The chow/sucrose-fed rats (b) escalated in sucrose intake while reducing prior chow intake ('anticipatory negative contrast'). Following the 15 sessions (in a, b), OSU6I62 (0, 5, 10, 15, and $30 \mathrm{mg} / \mathrm{kg}$ ) was tested for effects on food intake in the chow/chow (c) and the chow $/$ sucrose (d) group. Within each group, each rat received each dose and thus served as its own control. In the chow/chow-fed rats (c), OSU6I62 (I0 and 30 mg/kg) significantly reduced chow intake compared with vehicle upon analysis of both feeders together due to lack of a significant session $\times$ feeder interaction (repeated-measures ANOVA and within-subject contrast to vehicle). In the chow/sucrose-fed rats (d), significant effects of treatment compared with vehicle are displayed $(* p<0.05, * * p<0.01$, repeated-measures ANOVA and within-subject contrast to vehicle).

factor and day and feeder as within-subject factors. Significant main effects of diet were followed by repeatedmeasures ANOVA with day and feeder as within-subject factors for each diet group separately. Significant feeder $\times$ session interactions were followed by repeated-measures ANOVA within each feeder. A significant main effect of day was followed by Sidak post hoc to evaluate significant changes against day 1.

To detect overall effects of OSU6162 treatment in the binge-like eating model, food intake (kcal) was analyzed using repeated-measures ANOVA with diet group as the between-subject factor and feeder and treatment as within-subject factors. Upon confirmation of a significant main effect of diet, repeated-measures ANOVA with treatment and feeder as within-subject factors were performed for each diet group separately and significant feeder $\times$ treatment interactions were followed by repeatedmeasures ANOVA within each feeder. Significant main effect of treatment was followed by within-subject contrasts to vehicle.

Effects of systemic and intracerebral drug treatments on operant food seeking under FI1, FI15, and second-order schedules were analyzed using separate one-way repeatedmeasures ANOVAs for each drug and schedule as well as within each 15 min interval. Data of the FI15 and secondorder schedule were not normally distributed and therefore square root transformed before analysis. Note that the non- transformed number of lever presses is presented in the figures for the sake of clarity. Significant treatment effects were followed by within-subject contrasts to vehicle.

Statistical analyses were performed using the software SPSS (version 22, Chicago, IL) and all values were reported as mean \pm SEM. The significance level was set at $\alpha \leqslant 0.05$.

\section{RESULTS}

\section{Binge-like Eating Is Induced by Limited Access to Chocolate-Flavored Sucrose Pellets}

A binge-like eating pattern (ie, escalation of sucrose intake over time) was achieved by giving rats daily exposure to the following paradigm during 15 days of training: $2 \mathrm{~h}$ food deprivation, $10 \mathrm{~min}$ access to chow in the first feeder, followed by a $10 \mathrm{~min}$ access to either chow (chow/chow group) or chocolate-flavored sucrose pellets (chow/sucrose group) in the second feeder. There were significant overall main effects of diet group (ie, chow/chow $v s$ chow/sucrose; $\left.\mathrm{F}_{1,17}=118.3, p<0.001\right)$, session $\left(\mathrm{F}_{14,238}=18.6, p<0.001\right)$, and feeder $\left(\mathrm{F}_{1,17}=100.6, p<0.001\right)$, as well as a diet $\times$ session $\times$ feeder interaction $\left(\mathrm{F}_{14,238}=17.9, \quad p<0.001\right)$. Within-group analyses revealed significant session $\times$ feeder interactions in both diet groups (chow/chow: $\mathrm{F}_{14,112}=2.9$, $p<0.01$; chow/sucrose: $\mathrm{F}_{14,126}=42.5, p<0.001$ ). In the chow/chow group (Figure $2 \mathrm{a}$ ) there was a stable chow intake 
second-order
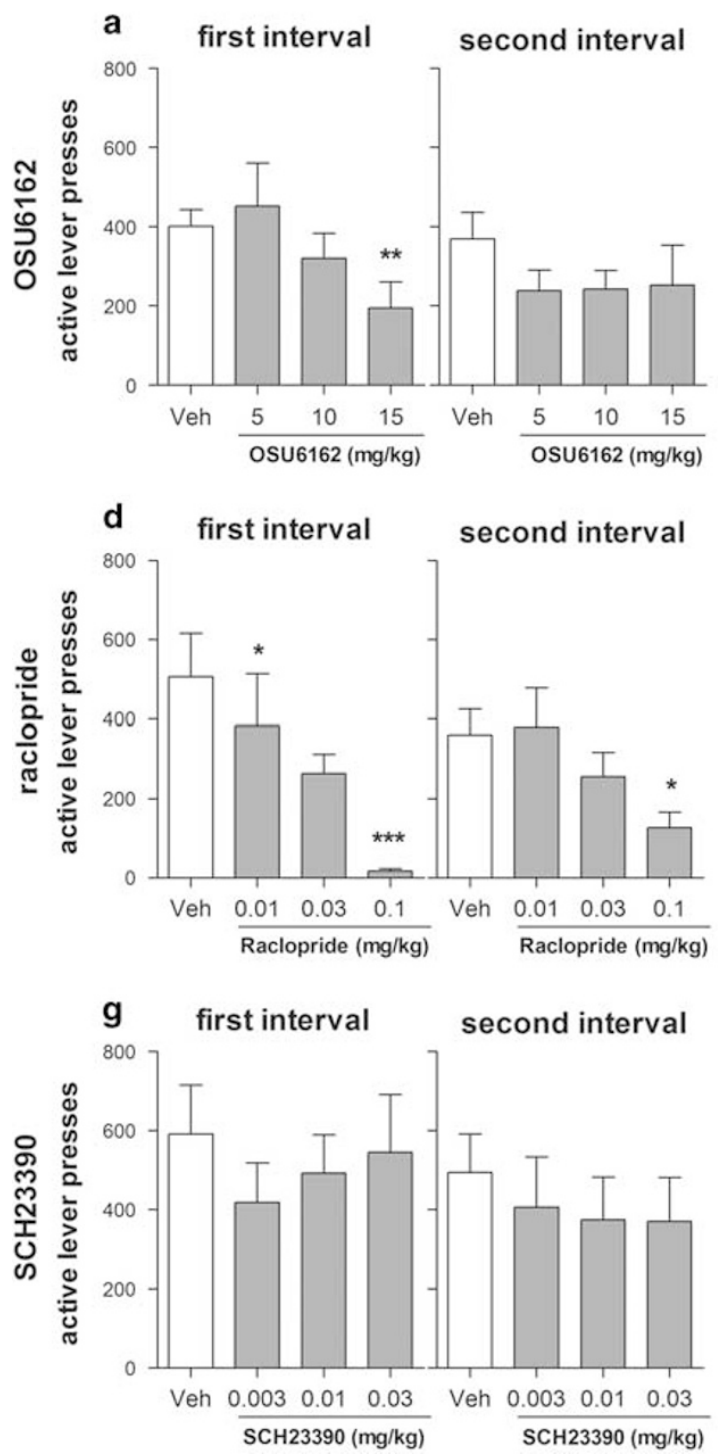

FI 15

FI 1
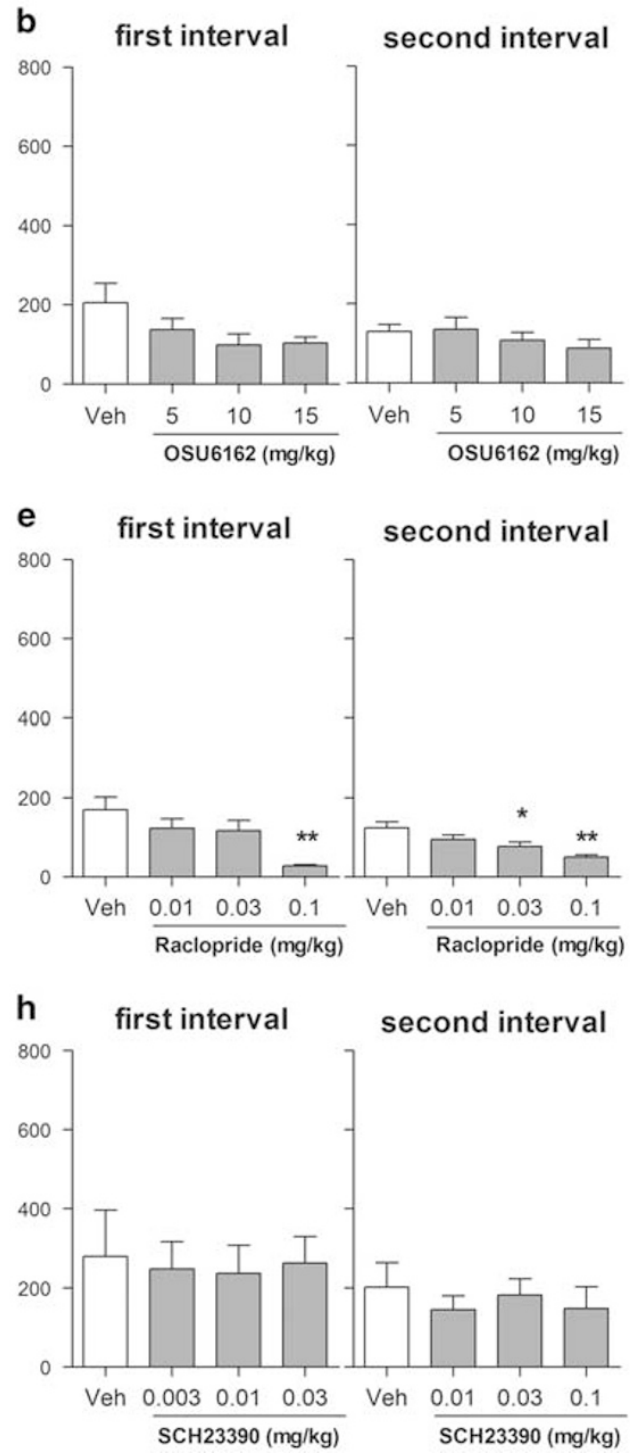

c

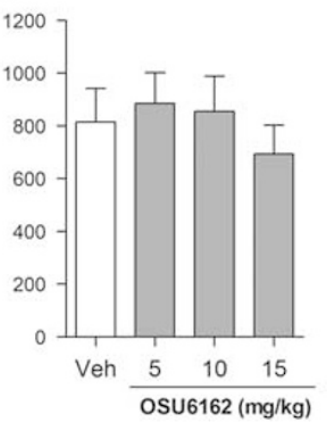

f

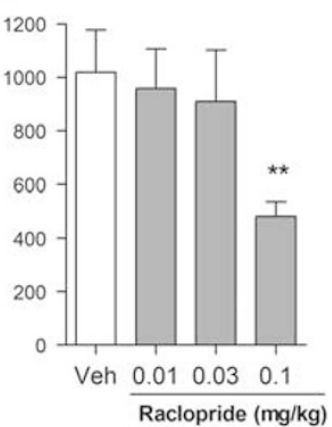

i

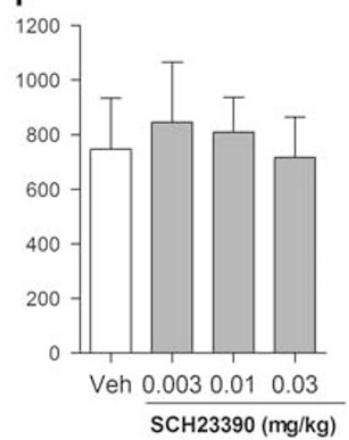

Figure 3 Effects of OSU6 I62, raclopride, and SCH23390 on seeking for highly palatable food under a fixed interval (FI) I and I5 min and a second-order schedule of reinforcement. Effects of OSU6I $62(0,5,10$, and I $5 \mathrm{mg} / \mathrm{kg}, \mathrm{a}-\mathrm{c})$, raclopride $(0,0.0 \mathrm{I}, 0.03,0.1 \mathrm{mg} / \mathrm{kg}, \mathrm{d}-\mathrm{f})$, and SCH23390 (0, $0.003,0.0 \mathrm{I}$, and $0.03 \mathrm{mg} / \mathrm{kg}, \mathrm{g}-\mathrm{i}$ ) ( $n=8 / \mathrm{group}$ ) on seeking for highly palatable food under a second-order (left panel) schedule and fixed interval schedules of I5 min (FII5, central panel) and I min (FII, right panel) of reinforcement are displayed. In both the second-order and FII 5 schedule, 20 pellets (chocolate-flavored sucrose) together with a 20 s conditioned stimuli (CS) were delivered at the end of each 15 min interval, leading to the first interval (before reward delivery) and second interval (after reward delivery). The second-order schedule differed from FII 5 by presentation of a contingent I s CS upon every tenth active lever presses during an interval. In the Fll schedule, I pellet and a 20 s CS were delivered upon an active lever press first after I min had elapsed, leading to 40 pellets delivered in total. Values represent mean \pm SEM number of active lever presses during each interval. Within each treatment group, each rat received each dose and thus served as its own control. Significant effects of treatment on active lever presses compared with vehicle are displayed (* $<<0.05$, *** $<0.0$ I, ***** $<0.00$ I, repeated-measures ANOVA and within-subject contrast to vehicle).

in both feeders during the 15 sessions. The post hoc analysis revealed that the chow/sucrose group significantly increased the sucrose consumption to binge-like levels on each session compared with the first session (Figure $2 \mathrm{~b}$ ) and significantly decreased the chow intake starting from session 6 , ie, they displayed an anticipatory negative contrast effect, undereating the first presented food in expectation of access to the more highly preferred chocolate-flavored sucrose pellets (Giuliano et al, 2012). Sucrose intake was similar to the binge-like levels previously reported in female
Lister Hooded (Giuliano et al, 2012) and Wistar rats (Cottone et al, 2008).

\section{OSU6162 Reduced Binge-Like Eating of Chocolate- Flavored Sucrose Pellets}

OSU6162 significantly reduced binge-like intake of chocolate-flavored sucrose pellets, but did not significantly affect the negative anticipatory contrast.

The effect of OSU6162 (5, 10, 15, and $30 \mathrm{mg} / \mathrm{kg}$, s.c.) on binge-like eating was evaluated after 15 sessions in the 
binge-eating paradigm. OSU6162 treatment significantly affected food intake, as indicated by a significant main effect of treatment $\left(\mathrm{F}_{4,48}=16.2, p<0.001\right)$, and also affected food intake differently in each diet group, depending on the feeder, as indicated by significant main effects of diet group $\left(\mathrm{F}_{1,17}=227.8, \quad p<0.001\right), \quad$ feeder $\quad\left(\mathrm{F}_{1,17}=144.5\right.$, $p<0.001)$, and a diet group $\times$ treatment $\times$ feeder interaction $\left(\mathrm{F}_{3.3,56.8}=16.5, \quad p<0.001\right)$. In the chow/chow group (Figure 2c), there was a significant main effect of treatment $\left(\mathrm{F}_{4,32}=7.7, p<0.001\right)$ but no feeder $\times$ treatment interaction $\left(\mathrm{F}_{4,32}=0.9, \mathrm{NS}\right)$. Hence, analyzing both feeders together, within-subject contrast to vehicle revealed that OSU6162 at $10 \quad(p<0.05)$ and $30 \mathrm{mg} / \mathrm{kg} \quad(p<0.001)$ significantly reduced chow intake. In the chow/sucrose group (Figure 2d), there was a significant main effect of treatment $\left(\mathrm{F}_{4,36}=9.7, p<0.001\right)$ and a significant feeder $\times$ treatment interaction $\quad\left(\mathrm{F}_{2.1,19.1}=22.9, \quad p<0.001\right)$. Within-subject contrasts to vehicle for each feeder revealed that 15 and $30 \mathrm{mg} / \mathrm{kg}$ OSU6162 significantly reduced sucrose, but did not significantly affect chow intake. Hence, there was no significant effect of OSU6162 on the anticipatory negative contrast.

\section{OSU6162 Reduced Cue-Controlled Seeking for Chocolate-Flavored Sucrose Pellets}

The effects of OSU6162 (5, 10, and $15 \mathrm{mg} / \mathrm{kg}$, s.c.), raclopride (0.01, 0.03, and $0.1 \mathrm{mg} / \mathrm{kg}$, s.c.), and SCH23390 (0.003, 0.01 , and $0.03 \mathrm{mg} / \mathrm{kg}$, i.p.) were evaluated on seeking behavior motivated by chocolate-flavored sucrose pellets under the second-order schedule of reinforcement and during prior training stages (FI1 and FI15).

OSU6162 treatment significantly affected seeking behavior under the second-order schedule (Figure 3a) during the first interval (before sucrose ingestion) $\left(\mathrm{F}_{3,21}=4.1, p<0.05\right)$, but not during the second interval (after sucrose ingestion) $\left(\mathrm{F}_{3,21}=1.1, \mathrm{NS}\right)$. Within-subject contrasts to vehicle revealed that OSU6162 $(15 \mathrm{mg} / \mathrm{kg})$ significantly reduced the responding on the active lever during the first interval only, before delivery of food. Under the FI15 schedule (Figure 3b), OSU6162 treatment produced an overall main effect during the first $\left(\mathrm{F}_{3,21}=3.3, p<0.05\right)$ but not the second interval $\left(\mathrm{F}_{3,21}=1.5\right.$, NS). However, within-subject contrast to vehicle revealed no significant effects of any dose. Seeking under FI1 was not affected by OSU6162 (Figure $3 c, \mathrm{~F}_{3,21}=2.0$, NS).

The D2-receptor antagonist raclopride significantly reduced sucrose-seeking behavior under the second-order a

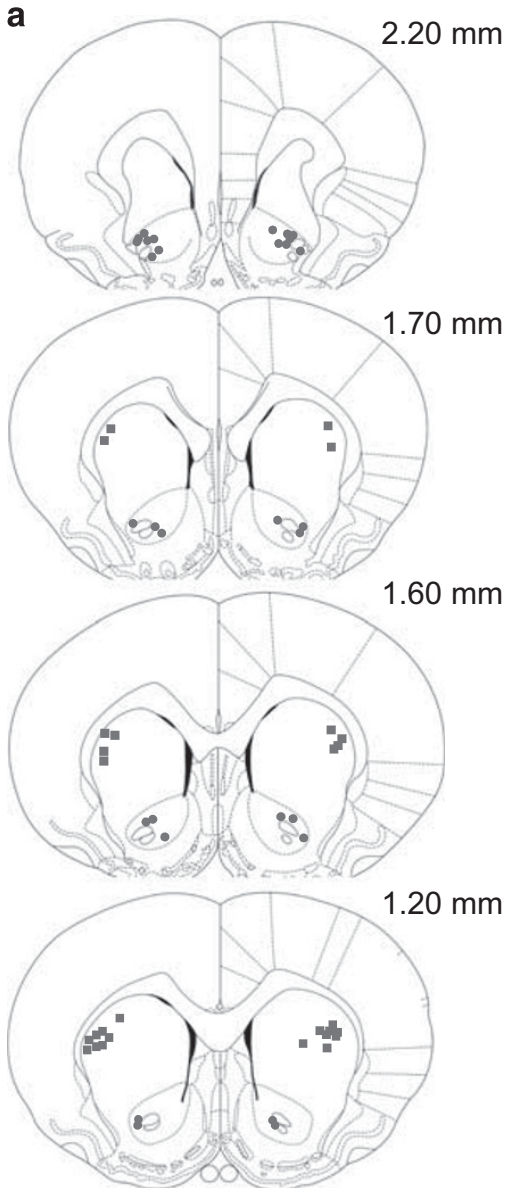

b
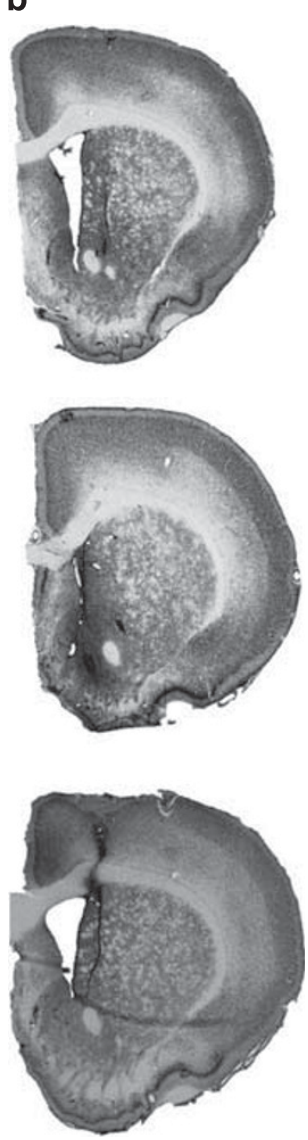

C
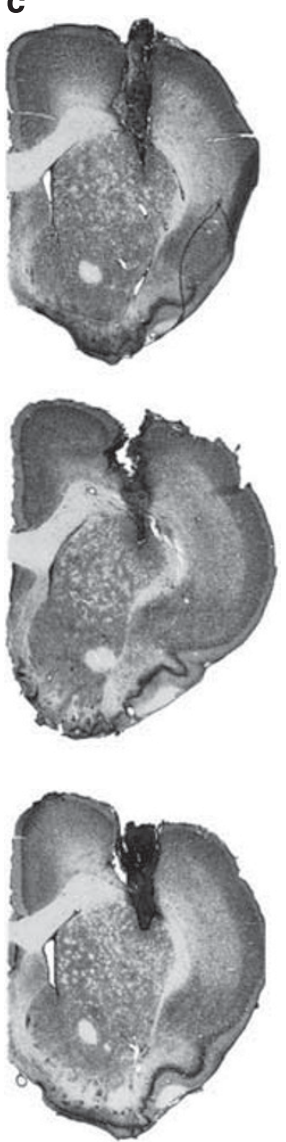

Figure 4 Histological illustration of locations of infusions into nucleus accumbens core and dorsolateral striatum. Guide cannulas were implanted above the nucleus accumbens (NAc, $n=15$ ) core or dorsolateral striatum (DLS, $n=14$ ). Coronal sections from +2.2 to $+1.2 \mathrm{~mm}$ anteroposterior from bregma represent histological verified locations of infusions into NAc core (filled circles) and DLS (filled squares) (a). Representative photomicrographs of Cresyl violet-stained coronal sections of NAc core- (b) and DLS- (c) cannulated rat brains, showing the guide cannula damage above and the infusion track into the targeted areas. 
a

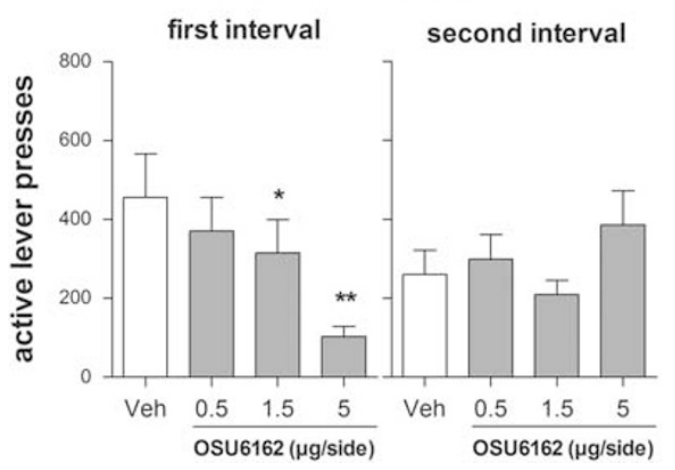

b

Dorsolateral Striatum

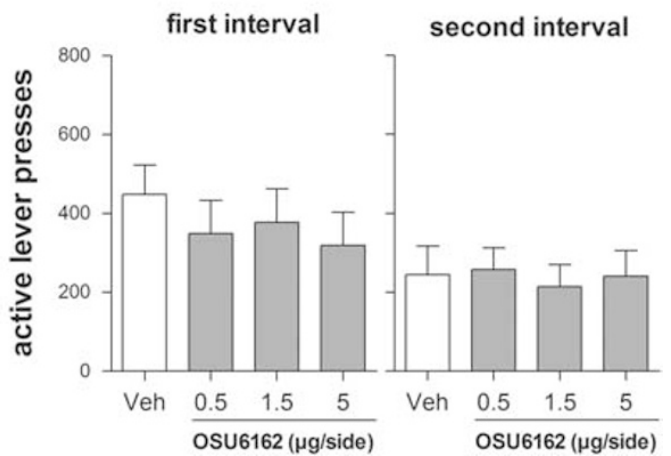

Figure 5 The role of the ventral and dorsal striatum in the effects of OSU6I62 on seeking for highly palatable food under a second-order schedule of reinforcement. Effects of OSU6I $62(0,0.5,1.5$, and $5 \mathrm{~kg} /$ side) infused into either the (a) nucleus accumbens (NAc) core $(n=15)$ or (b) dorsolateral striatum (DLS, $n=14$ ) on seeking for highly palatable food under a second-order schedule of reinforcement are displayed. At the end of each I 5 min interval, 20 pellets (chocolate-flavored sucrose) together with a $20 \mathrm{~s}$ conditioned stimuli (CS) were delivered, leading to the first interval (before reward delivery) and second interval (after reward delivery). During each interval, contingent I s CS were presented upon every tenth active lever press. Values represent mean \pm SEM number of active lever presses during each interval. Within each treatment group, each rat received each dose and thus served as its own control. Significant effects of treatment on active lever presses compared with vehicle are displayed $(* p<0.05, * * p<0.01$, repeated-measures ANOVA and within-subject contrast to vehicle).

(Figure 3d, first interval: $\mathrm{F}_{1.4,10.5}=15.5, p<0.01$; second interval: $\mathrm{F}_{3,21}=4.8, p<0.05$ ), the FI15 (Figure $3 \mathrm{e}$, first interval: $\mathrm{F}_{3,21}=12.9, p<0.001$; second interval: $\mathrm{F}_{3,21}=8.4$, $p<0.01$ ), and the FI1 (Figure 3f, $\mathrm{F}_{3,21}=5.1, p<0.01$ ) schedules. Within-subject contrasts to vehicle revealed that raclopride at the highest dose $(0.1 \mathrm{mg} / \mathrm{kg})$ significantly reduced lever pressing during all schedules and both intervals. Raclopride $0.01 \mathrm{mg} / \mathrm{kg}$ significantly reduced responding in the first interval of the second-order schedule compared with vehicle and at a dose of $0.03 \mathrm{mg} / \mathrm{kg}$ significantly reduced lever pressing during the second interval of the FI15 schedule.

SCH23390 treatment did not affect seeking behavior under either the second-order (Figure $3 \mathrm{~g}$, first interval: $\mathrm{F}_{3,21}=1.0$, NS; second interval: $F_{3,21}=0.9$, NS), the FI15 (Figure $3 \mathrm{~h}$, first interval: $F_{3,21}=0.2$, NS; second interval: $F_{3,21}=1.7, N S$ ), or the FI1 (Figure $3 \mathrm{i}, \mathrm{F}_{3,21}=0.7, \mathrm{NS}$ ) schedule.

None of the evaluated compounds affected pellet consumption in any schedule compared with vehicle; all rats consumed all pellets at the end of each interval during testing.

\section{Effects of Local Infusion of OSU6162 into the NAc Core or DLS on Cue-Controlled Seeking of Chocolate- Flavored Sucrose Pellets}

To evaluate the involvement of the NAc core and DLS in the effects of OSU6162 on cue-controlled sucrose seeking under a second-order schedule of reinforcement, OSU6162 (0.5, 1.5 , and $5 \mu \mathrm{g} /$ side) was bilaterally infused in either brain area before testing (Figure 4). OSU6162 infused into the NAc core (Figure $5 \mathrm{a}, n=15$ ) reduced responding for sucrose in the first (before sucrose ingestion) $\left(\mathrm{F}_{3,42}=9.2, p<0.001\right)$ but not in the second interval (after sucrose ingestion) $\left(\mathrm{F}_{3,42}=1.4, \mathrm{NS}\right)$. Within-subject contrasts to vehicle revealed that animals treated with OSU6162 at 1.5 and $5 \mu \mathrm{g} /$ side significantly reduced active lever pressing in the first interval. In contrast, OSU6162 infused into the DLS (Figure 5b, $n=14$ ), had no effect on responding for food in either the first $\left(F_{3,39}=1.4\right.$, $\mathrm{NS})$ or the second interval $\left(\mathrm{F}_{3,39}=0.07, \mathrm{NS}\right)$.

\section{DISCUSSION}

The monoamine stabilizer OSU6162 reduced binge-like consumption of chocolate-flavored sucrose pellets. Moreover, systemic as well as local administration of OSU6162 into the NAc core, but not the DLS, reduced cue-controlled seeking behavior for these pellets under a second-order schedule of reinforcement. Thus, OSU6162 affects both consummatory and appetitive behaviors of relevance to BED.

OSU6162 reduced sucrose intake in rats that had been trained to consume binge-like quantities of chocolateflavored sucrose pellets during brief daily access in behavioral chambers. However, OSU6162 did not significantly alter chow intake from the first feeder (before sucrose access). These results suggest that the monoamine-stabilizing effects of OSU6162 suppress overeating of palatable food in a model of BED directly during consumption without affecting prior anticipation. Furthermore, we previously reported that another potential treatment for BED, the selective $\mu$-opioid receptor antagonist GSK1521498, decreased bingeing on chocolate-flavored sucrose pellets and increased prior chow intake, suggestive of a decreased anticipatory negative contrast (Giuliano et al, 2012). Hence, we suggested that GSK1521498 has a specific effect on the impact of the hedonic value of the palatable food (Giuliano et al, 2012), whereas we found no evidence for an effect on anticipatory negative contrast after OSU6162 treatment in the current study. Together, these findings could indicate that opioid mechanisms are more important than dopamine signaling in anticipatory negative contrast or that any dopaminergic influence on this phenomenon is not blocked by OSU6162.

OSU6162 reduced chow intake in the chow/chow group (control group for the sucrose bingeing rats) in contrast to our previous study where GSK1521498 did not produce this effect (Giuliano et al, 2012). This indicates that OSU6162 is not generally specific to palatable food and can reduce intake of bland food. This apparent discrepancy to the effects in the sucrose-bingeing rats might be because of the fact that rats in the chow/chow group consumed higher amounts of chow from the first feeder than rats in the chow/sucrose group. 
These results, together with the attenuation of sucrose bingelike eating, suggest that OSU6162 moderates intake only if the amounts of food consumed are relatively high, regardless of palatability. This suggestion is further supported by the finding that OSU6162 attenuated binge-like eating $(34 \pm 2 \mathrm{kcal}$ sucrose) but did not affect sucrose consumption in the seeking procedures ( 40 pellets $=0.15 \mathrm{kcal}$ ). A similar selective, decreasing effect of OSU6162 only when the baseline is high, has previously been reported for locomotor activity (Natesan et al, 2006; Rung et al, 2008), alcohol intake (Steensland et al, 2012) and striatal L- $\left[{ }^{11} \mathrm{C}\right] \mathrm{DOPA}$ influx rate (Tedroff et al, 1998).

The mechanisms behind the monoamine-stabilizing effects of OSU6162 are not fully understood, but there are several plausible explanations for its effects on food intake in the present study. First, intake of palatable food, including sucrose, induces a peak in dopamine levels in the NAc, potentially coding for the motivational value of the food (Bassareo et al, 2002; Rada et al, 2005; Wilson et al, 1995). Thus, based on our previous finding showing that OSU6162 diminishes alcohol-induced NAc dopamine output in alcohol-naive rats (Steensland et al, 2012), we speculate that OSU6162 might attenuate this sucrose-induced dopamine peak, reducing the motivation to consume sucrose. Second, it is possible that an OSU6162-induced increase in dopaminergic tone before food presentation might underlie the reduction in food intake. This hypothesis is supported by rodent studies showing that: (1) OSU6162 slowly increases and maintains a stable elevated NAc dopamine output for several hours (Feltmann et al, 2016; Steensland et al, 2012); (2) optogenetically induced stable NAc dopamine release reduces voluntary sucrose intake (Mikhailova et al, 2016); (3) the dopamine-releasing compound lisdexamfetamine attenuates binge-like eating of chocolate (Vickers et $a l, 2015$ ); and (4) the dopaminereleasing compound methylphenidate reduces food consumption in humans and rodents (Davis et al, 2012; Thanos et al, 2015). Nevertheless, further studies are needed to elucidate the mechanism underlying the effect of OSU6162 to reduce food consumption.

In the cue-controlled sucrose-seeking (second-order schedule of reinforcement) procedure, OSU6162 significantly reduced responding during the first interval (before sucrose consumption), but not the second interval (after sucrose consumption). These results indicate that OSU6162 reduced incentive responding for the reward without affecting its hedonic value, consistent with the theory that the dopamine system is primarily involved in incentive motivation, but not hedonic aspects of food reward (Barbano and Cador, 2007; Berridge, 2007; Kelley et al, 2002). This effect is again in contrast to our previous findings with GSK1521498, which reduced seeking during both intervals (Giuliano et al, 2012), suggesting that $\mu$-receptor antagonism attenuates both the incentive motivational and hedonic impact of palatable food reward (for a review, see (Giuliano and Cottone, 2015).

The traditional dopamine D2 receptor antagonist raclopride, in contrast to OSU6162, reduced sucrose seeking under all schedules (FI1, FI15, and second-order), indicating that raclopride has a more general effect on seeking than OSU6162. These results indicate that although D2 dopamine receptors seem to be implicated in the effects of OSU6162 on cue-controlled sucrose seeking, the mechanism of action might differ between OSU6162 and raclopride. Accordingly, and in contrast to traditional D2 receptor antagonists, OSU6162 does not induce catalepsy at high (80\%) striatal D2/D3 occupancies in rodents (Natesan et al, 2006; Rung et al, 2008) or primates (Ekesbo et al, 1999). Moreover, the recent finding that OSU6162 occupies a maximum of $40 \%$ of striatal D2/D3 receptors in humans led to the hypothesis that OSU6162 might preferentially antagonize extrasynaptic, relative to synaptic, D2 receptors (Tolboom et al, 2014), potentially explaining the lack of extrapyramidal side effects (Johansson et al, 2012; Khemiri et al, 2015; Kloberg et al, 2014).

Intracranial infusion of OSU6162 into the NAc core recapitulated the systemic effects of the compound, resulting in decreased sucrose seeking under the second-order schedule of reinforcement during the first (pre-reward), but not second, interval. The NAc core is an important site mediating the capacity of food-associated conditioned reinforcers to control seeking behavior (Parkinson et al, 1999). Specifically, dopamine receptors within the NAc mediate sucrose seeking (Ikemoto and Panksepp, 1996), cueinduced reinstatement of food seeking (Floresco et al, 2008), and CS-dependent Pavlovian-Instrumental Transfer (Lex and Hauber, 2008). Whereas both systemic and local treatment with OSU6162 reduced seeking under the second-order schedule of reinforcement, none of the OSU6162 doses tested significantly reduced seeking under the FI15 schedule compared with vehicle, suggesting that OSU6162 interacts specifically with the conditioned reinforcing effects of food-associated cues. This might be particularly important in BED, where environmental cues such as commercials or smells often induce food cravings that are alleviated by seeking and ingestion of the food (Curtis and Davis, 2014; Sobik et al, 2005).

Infusions of OSU6162 into the DLS did not affect responding under the second-order schedule of reinforcement for sucrose, indicating that OSU6162 does not influence the stimulus-response mechanism located in this striatal domain. These results appear to be in contrast to previous studies showing that the mixed D1/D2 receptor antagonist $\alpha$-flupenthixol infused into the DLS reduced habitual cocaine seeking under this schedule (Belin and Everitt, 2008; Murray et al, 2012). This discrepancy might reflect a primary involvement of D1 signaling in the DLS, although this suggestion is contradicted by the present lack of effects of systemic SCH23390 administration. Alternatively, if there is a primary involvement of D2 signaling, this discrepancy could also reflect the suggested differences in the mechanisms of action of OSU6162 compared with traditional D2 receptor antagonists. Nevertheless, the involvement of the DLS and the effects of dopaminergic manipulations in the DLS on food seeking under the second-order schedule require further investigation.

In conclusion, the present study demonstrates that the monoamine stabilizer OSU6162 has the ability to reduce both binge-like eating and cue-controlled food seeking in rats. Together with a favorable side-effect profile in humans (Johansson et al, 2012; Khemiri et al, 2015; Kloberg et al, 2014), these results warrant further investigation of the potential of the monoamine stabilizer OSU6162 as a novel treatment for BED. 


\section{FUNDING AND DISCLOSURE}

We thank Dr Arvid Carlsson, Sahlgrenska Academy, University of Gothenburg, for generous donation of OSU6162 (supplied to Dr Carlsson by Pfizer Pharmaceuticals Inc). These studies were financially supported by a grant from the Swedish Society of Medicine (SLS-253061) to PS and JA, and a Medical Research Council Programme Grant (no. G1002231) to BJE. The Behavioural and Clinical Neuroscience Institute is cofunded by the Medical Research Council and the Welcome Trust. JA was supported by the Swedish Pharmaceutical Society and the Swedish Research Council (350-2012-230). A travel grant from the Swedish Society for Medical Research enabled KF to participate in this collaboration. PS was supported by the Swedish Research Council (2015-03525). The authors declare no conflict of interest.

\section{ACKNOWLEDGMENTS}

We thank Dr Natalia Arias and Maria Boulos for technical assistance.

\section{REFERENCES}

Avena NM (2007). Examining the addictive-like properties of binge eating using an animal model of sugar dependence. Exp Clin Psychopharmacol 15: 481-491.

Avena NM (2010). The study of food addiction using animal models of binge eating. Appetite 55: 734-737.

Barbano MF, Cador M (2007). Opioids for hedonic experience and dopamine to get ready for it. Psychopharmacology (Berl) 191: 497-506.

Bassareo V, De Luca MA, Di Chiara G (2002). Differential expression of motivational stimulus properties by dopamine in nucleus accumbens shell versus core and prefrontal cortex. $J$ Neurosci 22: 4709-4719.

Belin D, Everitt BJ (2008). Cocaine seeking habits depend upon dopamine-dependent serial connectivity linking the ventral with the dorsal striatum. Neuron 57: 432-441.

Bello NT, Hajnal A (2010). Dopamine and binge eating behaviors. Pharmacol Biochem Behav 97: 25-33.

Berridge KC (2007). The debate over dopamine's role in reward: the case for incentive salience. Psychopharmacology (Berl) 191: 391-431.

Cassin SE, von Ranson KM (2007). Is binge eating experienced as an addiction? Appetite 49: 687-690.

Colantuoni C, Schwenker J, McCarthy J, Rada P, Ladenheim B, Cadet JL et al (2001). Excessive sugar intake alters binding to dopamine and mu-opioid receptors in the brain. Neuroreport 12: 3549-3552.

Cottone P, Sabino V, Steardo L, Zorrilla EP (2008). Opioiddependent anticipatory negative contrast and binge-like eating in rats with limited access to highly preferred food. Neuropsychopharmacology 33: 524-535.

Curtis C, Davis C (2014). A qualitative study of binge eating and obesity from an addiction perspective. Eat Disord 22: 19-32.

Davis C, Fattore L, Kaplan AS, Carter JC, Levitan RD, Kennedy JL (2012). The suppression of appetite and food consumption by methylphenidate: the moderating effects of gender and weight status in healthy adults. Int J Neuropsychopharmacol 15: 181-187.

Davis H, Attia E (2017). Pharmacotherapy of eating disorders. Curr Opin Psychiatry 30: 452-457.

Di Ciano P (2008). Distinct contributions of dopamine receptors in the nucleus accumbens core or shell to established cocaine reinforcement under a second-order schedule. Eur Neuropsychopharmacol 18: 888-896.
Di Ciano P, Blaha CD, Phillips AG (1998). The relation between dopamine oxidation currents in the nucleus accumbens and conditioned increases in motor activity in rats following repeated administration of d-amphetamine or cocaine. Eur J Neurosci 10: 1113-1120.

Di Ciano P, Everitt BJ (2004). Conditioned reinforcing properties of stimuli paired with self-administered cocaine, heroin or sucrose: implications for the persistence of addictive behaviour. Neuropharmacology 47((Suppl 1)): 202-213.

du Hoffmann J, Nicola SM (2014). Dopamine invigorates reward seeking by promoting cue-evoked excitation in the nucleus accumbens. J Neurosci 34: 14349-14364.

Ekesbo A, Torstenson R, Hartvig P, Carlsson A, Sonesson C, Waters $\mathrm{N}$ et al (1999). Effects of the substituted (S)-3-phenylpiperidine (-)-OSU6162 on PET measurements of [11C]SCH23390 and [11C]raclopride binding in primate brains. Neuropharmacology 38: 331-338.

Ericson M, Blomqvist O, Engel JA, Soderpalm B (1998). Voluntary ethanol intake in the rat and the associated accumbal dopamine overflow are blocked by ventral tegmental mecamylamine. Eur J Pharmacol 358: 189-196.

Feltmann K, Fredriksson I, Wirf M, Schilstrom B, Steensland P (2016). The monoamine stabilizer (-)-OSU6162 counteracts downregulated dopamine output in the nucleus accumbens of long-term drinking Wistar rats. Addict Biol 21: 438-449.

Floresco SB, McLaughlin RJ, Haluk DM (2008). Opposing roles for the nucleus accumbens core and shell in cue-induced reinstatement of food-seeking behavior. Neuroscience 154: 877-884.

Giuliano C, Cottone P (2015). The role of the opioid system in binge eating disorder. CNS Spectr 20: 537-545.

Giuliano C, Robbins TW, Nathan PJ, Bullmore ET, Everitt BJ (2012). Inhibition of opioid transmission at the mu-opioid receptor prevents both food seeking and binge-like eating. Neuropsychopharmacology 37: 2643-2652.

Gonzales RA, Job MO, Doyon WM (2004). The role of mesolimbic dopamine in the development and maintenance of ethanol reinforcement. Pharmacol Ther 103: 121-146.

Ikemoto S, Panksepp J (1996). Dissociations between appetitive and consummatory responses by pharmacological manipulations of reward-relevant brain regions. Behav Neurosc 110: 331-345.

Ito R, Dalley JW, Howes SR, Robbins TW, Everitt BJ (2000). Dissociation in conditioned dopamine release in the nucleus accumbens core and shell in response to cocaine cues and during cocaine-seeking behavior in rats. J Neurosci 20: 7489-7495.

Johansson B, Carlsson A, Carlsson ML, Karlsson M, Nilsson MKL, Nordquist-Brandt E et al (2012). Placebo-controlled cross-over study of the monoaminergic stabiliser (-)-OSU6162 in mental fatigue following stroke or traumatic brain injury. Acta Neuropsych 24: 266-274.

Kelley AE, Bakshi VP, Haber SN, Steininger TL, Will MJ, Zhang M (2002). Opioid modulation of taste hedonics within the ventral striatum. Physiol Behav 76: 365-377.

Khemiri L, Steensland P, Guterstam J, Beck O, Carlsson A, Franck J et al (2015). The effects of the monoamine stabilizer (-)-OSU6162 on craving in alcohol dependent individuals: a human laboratory study. Eur Neuropsychopharmacol 25: 2240-2251.

Kloberg A, Constantinescu R, Nilsson MK, Carlsson ML, Carlsson A, Wahlstrom J et al (2014). Tolerability and efficacy of the monoaminergic stabilizer (-)-OSU6162 (PNU-96391A) in Huntington's disease: a double-blind cross-over study. Acta Neuropsych 26: 298-306.

Lex A, Hauber W (2008). Dopamine D1 and D2 receptors in the nucleus accumbens core and shell mediate Pavlovianinstrumental transfer. Learn Mem 15: 483-491.

Mikhailova MA, Bass CE, Grinevich VP, Chappell AM, Deal AL, Bonin KD et al (2016). Optogenetically-induced tonic dopamine 
release from VTA-nucleus accumbens projections inhibits reward consummatory behaviors. Neuroscience 333: 54-64.

Murray JE, Belin D, Everitt BJ (2012). Double dissociation of the dorsomedial and dorsolateral striatal control over the acquisition and performance of cocaine seeking. Neuropsychopharmacology 37: 2456-2466.

Naef L, Pitman KA, Borgland SL (2015). Mesolimbic dopamine and its neuromodulators in obesity and binge eating. CNS Spectr 20: 574-583.

Natesan S, Svensson KA, Reckless GE, Nobrega JN, Barlow KB, Johansson AM et al (2006). The dopamine stabilizers (S)-(-)-(3methanesulfonyl-phenyl)-1-propyl-piperidine [(-)-OSU6162] and 4-(3-methanesulfonylphenyl)-1-propyl-piperidine (ACR16) show high in vivo D2 receptor occupancy, antipsychotic-like efficacy, and low potential for motor side effects in the rat. J Pharmacol Exp Ther 318: 810-818.

Parkinson JA, Olmstead MC, Burns LH, Robbins TW, Everitt BJ (1999). Dissociation in effects of lesions of the nucleus accumbens core and shell on appetitive pavlovian approach behavior and the potentiation of conditioned reinforcement and locomotor activity by D-amphetamine. J Neurosci 19: 2401-2411.

Rada P, Avena NM, Hoebel BG (2005). Daily bingeing on sugar repeatedly releases dopamine in the accumbens shell. Neuroscience 134: 737-744.

Rung JP, Rung E, Helgeson L, Johansson AM, Svensson K, Carlsson A et al (2008). Effects of (-)-OSU6162 and ACR16 on motor activity in rats, indicating a unique mechanism of dopaminergic stabilization. J Neural Transm 115: 899-908.

Schienle A, Schafer A, Hermann A, Vaitl D (2009). Binge-eating disorder: reward sensitivity and brain activation to images of food. Biol Psychiatry 65: 654-661.

Sobik L, Hutchison K, Craighead L (2005). Cue-elicited craving for food: a fresh approach to the study of binge eating. Appetite 44: 253-261.

Sonesson C, Lin CH, Hansson L, Waters N, Svensson K, Carlsson A et al (1994). Substituted (S)-phenylpiperidines and rigid congeners as preferential dopamine autoreceptor antagonists: synthesis and structure-activity relationships. J Med Chem 37: 2735-2753.

Steensland P, Fredriksson I, Holst S, Feltmann K, Franck J, Schilstrom $B$ et al (2012). The monoamine stabilizer (-)-OSU6162 attenuates voluntary ethanol intake and ethanol-induced dopamine output in nucleus accumbens. Biol Psychiatry 72: 823-831.
Tedroff J, Torstenson R, Hartvig P, Sonesson C, Waters N, Carlsson A et al (1998). Effects of the substituted (S)-3-phenylpiperidine (-)-OSU6162 on PET measurements in subhuman primates: evidence for tone-dependent normalization of striatal dopaminergic activity. Synapse 28: 280-287.

Thanos PK, Robison LS, Steier J, Hwang YF, Cooper T, Swanson JM et al (2015). A pharmacokinetic model of oral methylphenidate in the rat and effects on behavior. Pharmacol Biochem Behav 131: 143-153.

Tolboom N, Berendse HW, Leysen JE, Yaqub M, van Berckel BN, Schuit RC et al (2014). The dopamine stabilizer (-)-OSU6162 occupies a subpopulation of striatal dopamine D2/D3 receptors: an [C]Raclopride PET Study in healthy human subjects. Neuropsychopharmacology 40: 472-479.

Vickers SP, Hackett D, Murray F, Hutson PH, Heal DJ (2015). Effects of lisdexamfetamine in a rat model of binge-eating. $J$ Psychopharmacol 29: 1290-1307.

Volkow ND, Wang GJ, Telang F, Fowler JS, Logan J, Childress AR et al (2006). Cocaine cues and dopamine in dorsal striatum: mechanism of craving in cocaine addiction. I Neurosci 26: 6583-6588.

Wang GJ, Geliebter A, Volkow ND, Telang FW, Logan J, Jayne MC et al (2011). Enhanced striatal dopamine release during food stimulation in binge eating disorder. Obesity (Silver Spring) 19: 1601-1608.

Wilson C, Nomikos GG, Collu M, Fibiger HC (1995). Dopaminergic correlates of motivated behavior: importance of drive. $J$ Neurosci 15(7 Pt 2): 5169-5178.

Wise RA (2006). Role of brain dopamine in food reward and reinforcement. Philos Trans R Soc Lond B Biol Sci 361: 1149-1158.

(c) (i) (2) $\odot$ This work is licensed under a Creative Commons Attribution-NonCommercial-NoDerivs $\quad 4.0$ International License. The images or other third party material in this article are included in the article's Creative Commons license, unless indicated otherwise in the credit line; if the material is not included under the Creative Commons license, users will need to obtain permission from the license holder to reproduce the material. To view a copy of this license, visit http://creativecommons.org/licenses/by-nc-nd/4.0/

(C) The Author(s) 2018

Supplementary Information accompanies the paper on the Neuropsychopharmacology website (http://www.nature.com/npp) 